\title{
PLANE WAVES WITH NEGATIVE PHASE VELOCITY IN ISOTROPIC CHIRAL MEDIUMS
}

\author{
Tom G. Mackay \\ School of Mathematics, University of Edinburgh \\ James Clerk Maxwell Building, King's Buildings, Edinburgh EH9 3JZ, United Kingdom
}

\begin{abstract}
The propagation of plane waves in an isotropic chiral medium (ICM) is investigated. Simple conditions are derived - in terms of the constitutive parameters of the ICM - for the phase velocity to be directed opposite to the direction of power flow. It is demonstrated that phase velocity and power flow may be oppositely directed provided that the magnetoelectric coupling is sufficiently strong.
\end{abstract}

Keywords: Negative phase velocity; isotropic chiral medium; negative refraction

\section{INTRODUCTION}

Homogeneous mediums in which plane waves propagate with phase velocity directed opposite to the direction of power flow are known as negative phase velocity (NPV) mediums. The archetypal NPV medium is the lossless, isotropic, dielectric-magnetic medium, characterized by the scalar permittivity $\epsilon$ and scalar permeability $\mu$, with $\epsilon<0$ and $\mu<0$ being satisfied simultaneously. In the 1960's Veselago predicted that such a medium would exhibit a range of interesting and potentially useful electromagnetic phenomenons, such as negative refraction, inverse Doppler shift and inverse C̆erenkov radiation [1]. Recent experimental observations involving the microwave illumination of certain composite metamaterials are supportive Veselago's predictions [2]-[5] and have sparked an intensification of interest in this area [6].

In reality, isotropic dielectric-magnetic mediums are characterized by complex-valued constitutive parameters $\epsilon$ and $\mu$. Phase velocity and power flow are oppositely directed in such a 
medium provided that the inequality [7]

$$
\frac{\operatorname{Re}\{\epsilon\}}{\operatorname{Im}\{\epsilon\}}+\frac{\operatorname{Re}\{\mu\}}{\operatorname{Im}\{\mu\}}<0
$$

is satisfied at a given frequency, where $\operatorname{Re}\{\bullet\}$ and $\operatorname{Im}\{\bullet\}$ denote the real and imaginary parts, respectively. Greater scope for NPV propagation is provided by more complex mediums, as has been demonstrated elsewhere for anisotropic [8, 9] and bianisotropic [10] mediums. The present communication concerns the generalization of the NPV condition (1), within the regime of isotropic mediums. Specifically, we consider the propagation of plane waves in the most general linear isotropic medium, namely the isotropic chiral medium (ICM). It is shown that the introduction of magnetoelectric coupling extends the possibilities for NPV planewave propagation in isotropic mediums.

\section{ANALYSIS}

Let us consider the propagation of planewaves with field phasors ${ }^{1}$

$$
\left.\begin{array}{l}
\mathbf{E}(\mathbf{r})=\mathbf{E}_{0} \exp (i k \hat{\mathbf{k}} \cdot \mathbf{r}) \\
\mathbf{H}(\mathbf{r})=\mathbf{H}_{0} \exp (i k \hat{\mathbf{k}} \cdot \mathbf{r})
\end{array}\right\},
$$

where $|\hat{\mathbf{k}}|=1$, in the isotropic chiral medium (ICM) described by the Tellegen constitutive relations [11]

$$
\left.\begin{array}{l}
\mathbf{D}(\mathbf{r})=\epsilon \mathbf{E}(\mathbf{r})+i \xi \mathbf{H}(\mathbf{r}) \\
\mathbf{B}(\mathbf{r})=-i \xi \mathbf{E}(\mathbf{r})+\mu \mathbf{H}(\mathbf{r})
\end{array}\right\} .
$$

The scalar constitutive parameters $\epsilon, \xi$ and $\mu$ are complex-valued in general, but for a lossless ICM we have $\epsilon, \mu, \xi \in \mathbb{R}$.

The wavenumbers $k=k_{R}+i k_{I}$, with $k_{R}, k_{I} \in \mathbb{R}$, are calculated from the planewave dispersion relation

$$
\operatorname{det}[\underline{\underline{L}}(i k \hat{\mathbf{k}})]=0
$$

which arises from the vector Helmholtz equation

$$
\underline{\underline{L}}(\nabla) \cdot \mathbf{E}_{0}=\mathbf{0},
$$

\footnotetext{
${ }^{1}$ Vectors are displayed in boldface, with the symbol^ ${ }^{\wedge}$ denoting a unit vector.
} 
wherein $\underline{\underline{L}}$ is the $3 \times 3$ dyadic differential operator defined as

$$
\underline{\underline{L}}(\nabla)=\nabla \times \nabla \times \underline{\underline{I}}-2 \omega \xi \nabla \times \underline{\underline{I}}-\omega^{2}\left(\epsilon \mu-\xi^{2}\right)
$$

The four $k$-roots of the quartic dispersion relation (4) are extracted as $k=k^{(i)}, k^{(i i)}, k^{(i i i)}$ and $k^{(i v)}$, where

$$
\left.\begin{array}{l}
k^{(i)}=-\omega(\sqrt{\epsilon \mu}+\xi) \\
k^{(i i)}=\omega(\sqrt{\epsilon \mu}-\xi) \\
k^{(i i i)}=-\omega(\sqrt{\epsilon \mu}-\xi) \\
k^{(i v)}=\omega(\sqrt{\epsilon \mu}+\xi)
\end{array}\right\}
$$

The rate of energy flow is provided by the time-averaged Poynting vector $\mathbf{P}=\frac{1}{2} \operatorname{Re}\left\{\mathbf{E} \times \mathbf{H}^{*}\right\}$, where the superscript ${ }^{*}$ indicates the complex conjugate. Utilizing the source-free Maxwell curl postulates, we find that for plane waves (2) propagating in the ICM specified by (3),

$$
\mathbf{P}=\frac{1}{2} \exp \left(-2 k_{I} \hat{\mathbf{k}} \cdot \mathbf{r}\right) \operatorname{Re}\left\{\frac{1}{\mu^{*}}\left[\mathbf{E}_{0} \times\left(\frac{k^{*}}{\omega} \hat{\mathbf{k}} \times \mathbf{E}_{0}^{*}-i \xi^{*} \mathbf{E}_{0}^{*}\right)\right]\right\}
$$

Our particular interest in this communication lies in the relative orientations of the phase velocity and the power flow, as provided by the relative orientations of $k_{R} \hat{\mathbf{k}}$ and $\mathbf{P}$. To pursue this matter, let us express the components of the electric field phasor as $\mathbf{E}_{0}=\left(E_{0 x}, E_{0 y}, E_{0 z}\right)$ and - without loss of generality - choose $\hat{\mathbf{k}}=\hat{\mathbf{z}}$. Thereby, it follows straightforwardly that

$$
k_{R} \hat{\mathbf{k}} \cdot \mathbf{P}=\frac{k_{R}}{2}\left|E_{0 y}\right|^{2} \exp \left(-2 k_{I} \hat{\mathbf{k}} \cdot \mathbf{r}\right) \operatorname{Re}\left\{\frac{1}{\mu^{*}}\left[\frac{k^{*}}{\omega}\left(1+|\alpha|^{2}\right)-i \xi^{*}\left(\alpha-\alpha^{*}\right)\right]\right\},
$$

where the complex-valued quotient

$$
\alpha=\frac{E_{0 x}}{E_{0 y}}
$$

has been introduced. The vector Helmholtz equation (5) yields

$$
\alpha=-\frac{[\underline{\underline{L}}(i k \hat{\mathbf{k}})]_{12}}{[\underline{\underline{L}}(i k \hat{\mathbf{k}})]_{11}}
$$

thus, the following evaluations of $\alpha$ are delivered for the wavenumbers (7)

$$
\alpha=\left\{\begin{array}{lrr}
i & \text { for } & k=k^{(i),(i i)} \\
-i & \text { for } & k=k^{(i i i),(i v)}
\end{array}\right.
$$


Upon combining (9) with (12) we find that

$$
k_{R} \hat{\mathbf{k}} \cdot \mathbf{P}= \begin{cases}\omega\left|E_{0 y}\right|^{2} \exp \left(-2 k_{I} \hat{\mathbf{k}} \cdot \mathbf{r}\right) \operatorname{Re}\{\sqrt{\epsilon \mu}+\xi\} \times \operatorname{Re}\left\{\sqrt{\frac{\epsilon}{\mu}}\right\} & \text { for } \quad k=k^{(i),(i v)} \\ \omega\left|E_{0 y}\right|^{2} \exp \left(-2 k_{I} \hat{\mathbf{k}} \cdot \mathbf{r}\right) \operatorname{Re}\{\sqrt{\epsilon \mu}-\xi\} \times \operatorname{Re}\left\{\sqrt{\frac{\epsilon}{\mu}}\right\} & \text { for } \quad k=k^{(i i),(i i i)}\end{cases}
$$

Therefore, NPV propagation occurs provided that the following inequalities are satisfied

$$
\begin{aligned}
& \operatorname{Re}\{\sqrt{\epsilon \mu}+\xi\} \times \operatorname{Re}\left\{\sqrt{\frac{\epsilon}{\mu}}\right\}<0 \quad \text { for } \quad k=k^{(i),(i v)} \\
& \left.\operatorname{Re}\{\sqrt{\epsilon \mu}-\xi\} \times \operatorname{Re}\left\{\sqrt{\frac{\epsilon}{\mu}}\right\}<0 \quad \text { for } \quad k=k^{(i i),(i i i)}\right\} .
\end{aligned}
$$

Finally, for a lossless ICM with $k \in \mathbb{R}$, we observe that the NPV conditions (14) reduce to

$$
\left.\begin{array}{l}
\sqrt{\epsilon \mu}<-\xi \quad \text { for } \quad k=k^{(i),(i v)} \\
\sqrt{\epsilon \mu}<\xi \quad \text { for } \quad k=k^{(i i),(i i i)}
\end{array}\right\} \text {. }
$$

\section{CONCLUDING REMARKS}

Through the introduction of magnetoelectric coupling, and the associated expansion of the constitutive parameter space, the scope for phase velocity and power flow to be oppositely directed is extended for isotropic mediums. In particular, it is demonstrated that if the magnitude of the magnetoelectric constitutive parameter $\xi$ is sufficiently large relative to the permittivity $\epsilon$ and permeability $\mu$ then NPV propagation is facilitated. While the parameter $\xi$ is typically small for naturally-occurring ICMs [12], the NPV potential for artificial ICMs, designed to provide enhanced magnetoelectric coupling, is highlighted by the results presented herein.

\section{References}

[1] V.G. Veselago, The electrodynamics of substances with simultaneously negative values of $\epsilon$ and $\mu$, Sov Phys Usp 10 (1968) 509-514.

[2] R.A. Shelby, D.R. Smith and S. Schultz, Experimental verification of a negative index of refraction, Science 292 (2001) 77-79. 
[3] A. Grbic and G.V. Eleftheriades, Experimental verification of backward--wave radiation from a negative index metamaterial, J Appl Phys 92 (2002), 5930-5935.

[4] C.G. Parazzoli, R.B. Greegor, K. Li, B.E.C. Koltenbah and M. Tanielian, Experimental verification and simulation of negative index of refraction using Snell's law, Phys Rev Lett $90(2003) 107401$.

[5] A.A. Houck, J.B. Brock and I.L. Chuang, Experimental observations of a left-handed material that obeys Snell's law, Phys Rev Lett 90 (2003), 137401.

[6] A. Lakhtakia and T.G. Mackay, Infinite phase velocity as the boundary between positive and negative phase velocities, Microwave Opt Technol Lett 20 (2004), 165-166.

[7] A. Lakhtakia, M.W. McCall and W.S. Weiglhofer, Negative phase-velocity mediums, In: W.S. Weiglhofer and A. Lakhtakia (eds), Introduction to complex mediums for electromagnetics and optics, SPIE Press, Bellingham, WA, USA, 2003.

[8] L. Hu and S.T. Chui, Characteristics of electromagnetic wave propagation in uniaxially anisotropic left-handed materials, Phys Rev B 66 (2002), 085108.

[9] M.K. Kärkkäinen, Numerical study of wave propagation in uniaxially anisotropic Lorentzian backward-wave slabs, Phys Rev E 68 (2003), 026602.

[10] T.G. Mackay and A. Lakhtakia, Plane waves with negative phase velocity in Faraday chiral mediums, Phys Rev E 69 (2004), 026602.

[11] A. Lakhtakia, Beltrami Fields in Chiral Media, World Scientific, Singapore, 1994.

[12] C.F. Bohren, Isotropic chiral materials, In: W.S. Weiglhofer and A. Lakhtakia (eds), Introduction to complex mediums for electromagnetics and optics, SPIE Press, Bellingham, WA, USA, 2003. 